\title{
ESTUDO PILOTO DE VALIDAÇÃO DO TESTE DE IDENTIFICAÇÃO DE SINAIS DE DISLEXIA (TISD)
}

\author{
Mirza Novaes Alves \\ Pedagoga, especialização em Neuropsicologia aplicada à Neurologia Infantil, Universidade Estadual \\ de Campinas (UNICAMP). \\ Ricardo Franco de Lima \\ Neuropsicólogo, mestre e doutorando em Ciências Médicas, Universidade Estadual de Campinas \\ (UNICAMP).
}

Rauni Jandé Roama Alves Psicólogo, mestre e doutorando em Psicologia, Pontifícia Universidade Católica de Campinas (PUCCampinas).

Cíntia Alves Salgado-Azoni Fonoaudióloga, pós-doutora em Ciências Médicas, UNICAMP.

Tatiana de Cássia Nakano Psicóloga, Professora Doutora do curso de pós-graduação Stricto Sensu em Psicologia, Pontifícia Universidade Católica de Campinas (PUC-Campinas).

Sylvia Maria Ciasca Neuropsicóloga, Professora Doutora Associada III, Departamento de Neurologia, UNICAMP.

\begin{abstract}
Resumo
O trabalho objetivou obter primeiras evidências de validade do Teste de Identificação de Sinais de Dislexia (TISD). Foram investigados dois grupos de crianças, um sem dificuldades de aprendizagem $(n=9)$ e outro com dificuldades de aprendizagem $(n=11)$, sendo o segundo selecionado em razão de possuir risco para transtornos de aprendizagem. Foram utilizados para coleta de dados o Teste de Desempenho Escolar (TDE) e TISD. Os resultados evidenciaram diferenças entre os grupos nos escores dos subtestes de Leitura, Escrita, Cálculo, Consciência Fonológica, Memória de trabalho e no Total, com melhor desempenho do grupo sem dificuldades de aprendizagem. Correlações significativas e negativas entre os subtestes do TDE e do TISD foram encontradas. Verificou-se que o TISD mostrou-se capaz de diferenciar os grupos, assim como obteve correlações com outro instrumento que avaliava construtos semelhantes, sendo o objetivo desse estudo alcançado.

Palavras-chave: neuropsicologia; avaliação psicológica; dislexia; dificuldades de aprendizagem.
\end{abstract}




\title{
VALIDATION PILOT STUDY OF THE IDENTIFYING SIGNS OF DYSLEXIA TEST (TISD)
}

\begin{abstract}
This study aimed to obtain the first evidence of validity to the Test Identification Signs of Dyslexia (TISD). We investigated two groups of children, one without learning difficulties $(n=9)$ and other with learning difficulties $(n=11)$; the second group was selected due to possible risk of learning disabilities. Two instruments were used for data collection Academic Performance Test (TDE) and TISD. The results showed differences between groups in the scores of the subtests of reading, writing, calculation, phonological awareness, working memory and total, with better performance in the group without learning difficulties. Significant and negatives correlations between TDE and TISD was found. It was found that the TISD proved capable of differentiating the groups, as well as correlations obtained with another instrument that assessed similar constructs, the aim of this study was reached.

Keywords: neuropsychology; psychological assessment; dyslexia; learning disabilities.
\end{abstract}

\section{ESTUdiO PILOTO DE LA VALIDACIÓN DE LA PRUEBA DE IDENTIFICACIÓN DE SEÑALES DE DISLEXIA (TISD)}

\begin{abstract}
Resumen
Este estudio tuvo como objetivo obtener la primera evidencia de la validez de la Prueba de Identificación de Señales de Dislexia (TISD). Se evaluaron dos grupos de niños, uno sin dificultades de aprendizaje $(n=9)$ y otro con dificultades de aprendizaje $(n=11)$; se seleccionó este segundo grupo por su posible riesgo de trastornos del aprendizaje. Se utilizaron tres instrumentos para recopilar datos: hoja de identificación del niño, Test de Rendimiento Académico (TDE) y TISD. Los resultados mostraron diferencias entre los grupos en las puntuaciones de los subtests de lectura, escritura, cálculo, conciencia fonológica, memoria de trabajo y total, con un mejor rendimiento en el grupo sin dificultades de aprendizaje. Correlaciones significativas y negativas entre TISD y TDE fueran fundadas. Se encontró que la TISD demostró ser capaz de diferenciar los grupos, así como correlaciones obtenidas con otro instrumento que evaluó construcciones similares, con esto, se alcanzó el objetivo del estudio.
\end{abstract}

Palabras clave: neuropsicología; evaluación psicológica; dislexia; problemas de aprendizaje.

\section{INTRODUÇÃO}

Desde 1968, quando os transtornos de aprendizagem foram classificados nos Estados Unidos como "condições debilitantes", tem sido cada vez mais observados progressos relacionados às questões acerca da sua classificação e definição (Fletcher, Lyons, Fuchs, \& Barnes, 2009), processos cognitivos envolvidos (Siegel, 2003), correlatos neurobiológicos (Eden \& Zeffiro, 1998; Shaywitz \& Shaywitz, 2005), aspectos genéticos (Grigorenko, 2005; Plomin \& Kovas, 2005), intervenções (Swanson, Harris, \& Graham, 2005) e práticas de avaliação (Fuchs \& Fuchs, 1998). 
A utilização do termo "transtorno de aprendizagem" (TA) implica em algumas precauções, uma vez que a confusão mais frequentemente observada diz respeito ao seu uso equivocado enquanto sinônimo de "dificuldades de aprendizagem" (Alves \& Nakano, no prelo; Carvalho, Crenitte, \& Ciasca, 2007). Convém esclarecer que um indivíduo com "dificuldades de aprendizagem" fará parte de um grupo de risco para TA, mas que o uso do termo "dificuldades de aprendizagem" apresenta caráter mais global, podendo envolver também alterações no processo de ensino e aprendizagem, falhas no processo de alfabetização, condições relacionadas a fatores psicossociais, assim como pode ser proveniente de diferentes alterações neurológicas e/ou genéticas, bem como deficiências física e intelectual (Germano \& Capellini, 2011; Lima, 2011). Especificamente, TA refere-se a um grupo de dificuldades, que possuem origem específica, decorrentes de disfunções no Sistema Nervoso Central (Wong \& Butler, 2012).

Os TA costumam envolver três grandes áreas de habilidades acadêmicas: matemática, escrita e leitura (Organização Mundial da Saúde, OMS, 2008), sendo que, em relação ao transtorno de leitura, foco do presente estudo, verificam-se os mais altos índices na população quando comparado aos demais transtornos (incidência de cerca de 4\%), sendo também comumente encontrado na literatura sob a nomenclatura de Dislexia do Desenvolvimento (DD) (American Psychological Association, APA, 2002).

Sinais indicativos para identificação da DD são apresentados em manuais diagnósticos (APA, 2002; OMS, 2008), cujos critérios envolvem: (a) frequentemente apresentam desenvolvimento tardio da fala; (b) a leitura oral é caracterizada por distorções, substituições ou omissões; (c) a leitura em voz alta e silenciosa é lenta e caracterizada por erros de compreensão; (d) as dificuldades não devem ser atribuídas à escolarização inadequada e comprometimentos sensoriais (as dificuldades devem exceder aqueles que podem estar associados); (e) é um comprometimento no desenvolvimento das habilidades da leitura (rendimento inferior ao esperado para a idade cronológica, para a inteligência medida, tanto em aspectos de correção quanto em velocidade e compreensão, medidos por testes padronizados).

Dada sua importância e a necessidade de identificação do transtorno, diferentes instrumentos que visam avaliar as habilidades cognitivo-linguísticas comprometidas nos TA, em especial, na Dislexia do Desenvolvimento, têm sido 
descritos na literatura internacional e nacional. Internacionalmente Cox (2002) elaborou o Test of Dyslexia and Dysgraphia (TODD) para auxiliar no diagnóstico da DD e disgrafia, por meio da avaliação da memória de símbolos, de palavras, habilidades fonológicas, processamento visual e nomeação rápida de símbolos. Nicolson e Fawcett (2003), por sua vez, elaboraram o Dyslexia Early Screening Test (DEST-2) que fornece valor indicativo de risco para possível transtorno de aprendizagem. Nele são avaliadas as competências para nomeação rápida, discriminação fonêmica, estabilidade postural, rima, dígitos, nomeação de dígitos, nomeação de letras, ordem de sons, cópia de formas, atenção, vocabulário, coordenação visomotora.

Por outro lado, para o contexto brasileiro são escassas as propostas de instrumentos que avaliem habilidades cognitivas e escolares que caracterizam a DD. Capovilla, Smythe, Capovilla e Everatt (2001) realizaram estudo preliminar para adaptação do International Dyslexia Test (IDT). Este instrumento avalia de maneira mais completa diferentes habilidades cognitivo-linguísticas e é composto por subtestes de leitura, escrita, habilidades matemáticas, consciência fonológica, processamento auditivo, discriminação fonológica, memória de curtoprazo, memória sequencial auditiva, processamento visual, discriminação e percepção visual, memória de curto-prazo visual, memória sequencial visual, velocidade de processamento, sequenciamento, habilidades motoras e raciocínio, sendo importante destacar o fato de que seus estudos de busca por evidências de validade para a realidade brasileira não foram finalizados. Posteriormente, Capellini et al. (2009) elaboraram protocolo para identificação precoce de problemas de leitura, composto pelos subtestes: conhecimento do alfabeto, consciência fonológica, memória de trabalho, velocidade de acesso à informação fonológica, atenção visual, leitura de palavras e não palavras e compreensão de frases a partir de figuras apresentadas, mas também não houve finalização de seus estudos por busca de evidências de validade.

Diante da lacuna existente no país em relação à identificação da DD, visando disponibilizar um novo instrumento para avaliação de problemas de aprendizagem nos contextos clínico e educacional, o Teste de Identificação de Sinais de Dislexia (TISD) foi construído (Alves, 2011). O TISD é composto por oito subtestes que se propõem a avaliar tanto habilidades cognitivas quanto acadêmicas, relacionadas a: (1) Leitura; (2) Escrita; (3) Atenção Visual; (4) Cálculo; (5) Habilidades Motoras; (6) Consciência fonológica; (7) Nomeação 
Rápida; (8) Memória de Trabalho. Seus itens foram elaborados tendo como base produções nacionais e testes internacionais para avaliação da DD, sendo selecionadas as habilidades mais comumente avaliadas em todos eles.

No entanto, a construção de um instrumento desse porte, que seja reconhecido cientificamente, não pode simplesmente limitar-se à construção de seus itens, considerado somente primeiro passo de um processo de construção de instrumento, e contemplado como um método de busca por evidências de validade baseadas no conteúdo do teste (Anastasi \& Urbina, 2000). Esse tipo de validade envolve ainda outros estudos, tais como: análises teóricas do construto, definição do construto e a análise do instrumento por juízes especialistas (Nunes \& Primi, 2010) - estudos esses também já realizados com o TISD (Alves, Lima, Salgado-Azoni, Carvalho, \& Ciasca, no prelo).

Existem mais quatro possíveis tipos de evidências de validade a serem investigadas durante o processo de construção de instrumento (APA, 1999): (a) evidências baseadas nas relações com variáveis externas, (b) evidências baseadas na estrutura interna, (c) evidências baseadas no processo de resposta e (d) evidências baseadas nas consequências de testagem. Considerando-se que o foco do estudo aqui apresentado baseia-se no primeiro tipo, referente aos estudos de investigação sobre a capacidade que um teste tem em predizer o desempenho de um sujeito, ou seja, se a medida realizada está relacionada com aquele comportamento apresentado em seu cotidiano, o presente trabalho teve como objetivo obter as primeiras evidências desse tipo de validade do TISD. Tal investigação ocorreu tomando-se a comparação do instrumental entre grupos de crianças com e sem dificuldades de aprendizagem, já que esse último caracteriza-se enquanto um grupo de risco para DD, e verificando-se a sua relação com um intrumento de desempenho escolar, que é composto por construtos semelhantes.

\section{MÉTODO}

\section{Participantes}

Participaram da pesquisa 20 crianças, de ambos os sexos ( 6 meninos e 14 meninas), com idade entre 10 e 11 anos $(M=10,55 ; D P=0,51)$, cursando o $4^{\circ}$ ou $5^{\circ}$ ano do Ensino Fundamental de uma escola pública, divididas em dois grupos:

(a) Grupo com queixas de dificuldades de aprendizagem (GD) ( $n=11$, sendo sete do sexo feminino e quatro do masculino, com idade média de 10,55 anos, 
$\mathrm{DP}=0,52$; dez delas matriculadas no quinto ano escolar e uma no quarto ano); e (b) Grupo controle sem queixas de dificuldades de aprendizagem (GC) $(n=9$, sendo sete do feminino e duas do sexo masculino, com idade média de 10,56, $\mathrm{DP}=0,53$; todas matriculadas no quinto ano escolar).

Diferentes análises foram conduzidas com o objetivo de verificar $a$ equiparação dos dois grupos em relação ao sexo do participante, idade e série escolar, sendo que os resultados demonstraram que não houve diferenças estatísticas significativas entre os grupos em relação ao sexo $(p=0,64$; Teste Exato de Fisher), idade ( $p=0,96$; Teste Mann-Whitney) e série escolar $(p=1,00$; Teste Exato de Fisher).

O critério para inclusão das crianças em cada um dos grupos envolveu a indicação dos professores: apresentar ou não dificuldades de aprendizagem em leitura e/ou escrita e/ou cálculo, sendo dessa forma composto ambos os grupos. Além disso, as crianças deveriam apresentar desempenho escolar dentro ou abaixo da média, conforme o grupo, avaliado pelo Teste de Desempenho Escolar (TDE) (Stein, 1994). Para a amostra geral, outros critérios de inclusão foram adotados: não apresentar histórico de doenças neurológicas; apresentar de acuidade visual e auditiva dentro dos padrões de normalidade, de acordo com o relato dos pais/responsáveis - esses critérios foram estabelecidos para que houvesse maior controle sobre os grupos, de forma que as dificuldades apresentadas pelas crianças não fossem decorrentes e explicáveis por alterações desses fatores, assim como no caso de crianças com DD. O critério de exclusão adotado foi o de desistência da crinça durante o processo avaliativo.

Materiais

Teste de Identificação de Sinais de Dislexia (TISD) (Alves, 2011)

Instrumento elaborado para avaliar sinais indicativos para a DD, destinado para crianças com faixa etária entre 8 e 11 anos e de aplicação individual, composto por oito subtestes (Tabela 1). Para os itens respondidos erroneamente somam-se pontos, de forma que quanto mais pontos o sujeito obtiver, pior terá sido seu desempenho no teste. A construção do instrumento ocorreu em duas etapas. Na primeira, que envolveu aspectos teóricos, foi realizada revisão da literatura para identificar habilidades cognitivo-linguísticas incluídas em instrumentos para avaliação da DD. Assim, todos os subtestes que compõem o TISD avaliam habilidades acadêmicas (leitura, escrita e cálculo) e cognitivas 
relacionadas à linguagem escrita (consciência fonológica, acesso ao léxico e memória de trabalho fonológica), conforme a revisão realizada. De modo específico, o subteste "Cálculo" foi incluído a fim de que, por meio de estudos de validação, seja verificado se o mesmo mostra-se discriminante na avaliação do grupo critério de DD, já que um dos critérios diagnósticos para esse transtorno é ter essa habilidade acadêmica preservada (Caldonazzo, Salgado, Capellini, \& Ciasca, 2006; OMS, 2008). Adicionalmente, o subteste "Habilidade Motora" foi incluído em função de algumas teorias e instrumentos sustentarem a hipótese de alteração dessa habilidade em indivíduos com DD (Nicolson \& Fawcett, 2011). Uma segunda etapa, construção do instrumento, contemplou o processo de composição dos itens, instruções, procedimentos de administração, elaboração do material estímulo, análise de juízes e construção da versão preliminar (Alves et al., no prelo).

Teste de Desempenho Escolar (TDE) (Stein, 1994)

Consiste em um instrumento que avalia as capacidades básicas para o desempenho escolar, nas áreas de escrita, aritmética e leitura, apontando quais áreas da aprendizagem escolar que estão preservadas ou prejudicadas. Este instrumento foi escolhido pelos seguintes motivos: (a) trata-se de um teste já validado e padronizado para a população brasileira; (b) fornece parâmetro para a diferenciação dos grupos; (c) pode ser usado para correlação com os subtestes do TISD. O TDE é composto por três subtestes: (1) Escrita: escrita de nome próprio e de palavras isoladas apresentadas, sob a forma de ditado. Constitui-se de uma lista de 45 palavras, que obedecem a uma ordem crescente de dificuldade ortográfica; (2) Aritmética: 38 itens compostos por problemas de solução oral e cálculos de operações aritméticas por escrito; (3) Leitura: reconhecimento de palavras isoladas do contexto. É constituído de 75 palavras em ordem crescente de dificuldade, obedecendo ao seguinte critério: gradação dos fonemas segundo as relações fonológico-ortográficas, número de sílabas, grau de familiaridade com o vocábulo e padrões silábicos. Cada item respondido corretamente recebe um ponto, de modo que pontuações maiores correspondem a melhores desempenhos. Os resultados são convertidos em uma escala, sendo classificados em: superior, médio e inferior para cada série escolar. 
Tabela 1.

Subtestes do TISD e suas pontuações.

\begin{tabular}{|c|c|c|}
\hline Subteste & Itens pontuados & $\begin{array}{c}\text { Pontuação máxima em } \\
\text { cada item e total }\end{array}$ \\
\hline \multirow{3}{*}{$\begin{array}{c}\text { Leitura } \\
\text { Reconhecimento de letras, } \\
\text { leitura de palavras e } \\
\text { pseudopalavras. }\end{array}$} & $\begin{array}{l}\text { L1- reconhecimento de } 21 \text { letras do } \\
\text { alfabeto }\end{array}$ & 21 \\
\hline & L2- leitura de nove palavras & \multirow{2}{*}{$\begin{array}{c}09 \\
\text { 09/ Total: } 39\end{array}$} \\
\hline & L3- Leitura de nove pseudopalavras & \\
\hline \multirow{3}{*}{$\begin{array}{l}\text { Escrita } \\
\text { Escrita de letras, de palavras e } \\
\text { pseudopalavras. }\end{array}$} & E1- ditado de 21 letras do alfabeto & 21 \\
\hline & E2- ditado de nove palavras & 09 \\
\hline & E3- ditado de nove pseudopalavras & 09/ Total: 39 \\
\hline \multicolumn{3}{|l|}{ Atenção visual } \\
\hline \multirow{2}{*}{$\begin{array}{l}195 \text { letras distribuídas } \\
\text { randomicamente e a criança } \\
\text { deve identificar a letra "p". }\end{array}$} & AV1- Total de adição & 176 \\
\hline & AV2-Total de omissão & 19/ Total: 195 \\
\hline \multirow{4}{*}{$\begin{array}{l}\text { Cálculo } \\
\text { Quatro problemas a serem } \\
\text { resolvidos mentalmente }\end{array}$} & C1- problema de adição & 01 \\
\hline & C2- problema de subtração & 01 \\
\hline & C3- problema de multiplicação & 01 \\
\hline & C4- problema de divisão & 01/ Total: 4 \\
\hline \multirow{5}{*}{$\begin{array}{l}\text { Habilidades motoras } \\
\text { Cópia de uma figura com linhas } \\
\text { e figuras geométricas. }\end{array}$} & HM1- cópia de círculo & 02 \\
\hline & HM2- cópia de retângulo & 02 \\
\hline & HM3- cópia de quadrado & 02 \\
\hline & HM4- cópia de triângulo & 02 \\
\hline & HM5- cópia da figura de linhas & 02/ Total: 10 \\
\hline \multirow{2}{*}{$\begin{array}{c}\text { Consciência fonológica } \\
\text { Identificação de rima e produção } \\
\text { de rima }\end{array}$} & CF1- identificação de três rimas & 03 \\
\hline & CF2- produção de três rimas & 03/ Total: 6 \\
\hline \multirow{2}{*}{$\begin{array}{c}\text { Nomeação rápida } \\
\text { Nomeação rápidade de letras e } \\
\text { números }\end{array}$} & NR1- conjunto de 25 letras & 25 \\
\hline & NR2- conjunto de 25 números & $25 /$ Total $50+$ tempo \\
\hline \multirow{2}{*}{$\begin{array}{l}\text { Memória de trabalho } \\
\text { Repetição de sequências de } \\
\text { dígitos e pseudopalavras. }\end{array}$} & MT1- sequências de dígitos & 06 \\
\hline & MT2- sequências de pseudopalavras & 06/ Total: 12 \\
\hline
\end{tabular}

\section{Procedimentos}

A presente pesquisa foi aprovada pelo Comitê de Ética em Pesquisa da FCM/UNICAMP (Parecer de no 946/2011). Para a realização do estudo, inicialmente foi entregue uma carta de apresentação para a escola, explicitando o objetivo da pesquisa para obtenção da autorização para o desenvolvimento da mesma. Em seguida, foi realizada reunião com os professores para apresentação dos objetivos da pesquisa e montagem dos grupos GD e GC. A partir da listagem dos alunos indicados, foi enviado aos pais/responsáveis dos mesmos, o TCLE solicitando autorização para participação de seu(sua) filho(a) na pesquisa, 
juntamente com a ficha de identificação. Após autorização dos pais/responsáveis, as crianças foram convocadas para avaliação individual, em período escolar, em sala destinada para essa finalidade, por aproximadamente duas sessões de trinta minutos cada. Após a coleta dos dados, esses foram corrigidos e tabulados, conforme plano de análise dos dados. Todos os resultados foram repassados aos pais, professoras envolvidas e corpo gestor da escola. Todos os alunos do GD foram encaminhados para avaliações interdisciplinares para a detecção de possíveis transtornos de aprendizagem.

Análise dos dados

Foi utilizada a planilha do programa IBM Statistical Package for Social Sciences (SPSS Statistics 20.0 for Windows $®$ ). Para a estatística inferencial foram usados testes não-paramétricos: para a comparação do desempenho dos grupos nos instrumentos foi utilizado o teste de Mann-Whitney (para variáveis numéricas). Esta análise foi complementada pelo cálculo da magnitude de efeito (Cohen's d), na qual: $d=0,20$ (pequeno), $d=0,50$ (médio) e $d=0,80$ (elevado) (Conboy, 2003). Resultados negativos são interpretados como pior desempenho no GD. Nos instrumentos nos quais escores superiores são interpretados como prejuízos (como o TISD) a interpretação é inversa; o teste Exato de Fisher foi usado para variáveis categóricas; para correlação entre os escores dos instrumentos foi utilizado o teste de correlação de Spearman. A magnitude do efeito foi considerada baixa $(<0,30)$ moderada $(0,40$ até 0,60$)$ e alta $(>0,70)$ (Dancey \& Reidy, 2006). O nível de significância adotado foi $\mathrm{p} \leq 0,05$.

\section{RESULTADOS}

Quando consideradas as classificações obtidas no TDE (Tabela 2), pode-se perceber que houve diferenças significativas entre os grupos GD e GC nas habilidades de Escrita $(p \leq 0,01)$ e Leitura $(p \leq 0,001)$, nas quais os participantes do grupo com queixa de dificuldades de aprendizagem apresentaram maior concentração de classificações consideradas "inferior" e os participantes do grupo controle na classificação "média". Quando os grupos são comparados em relação às médias obtidas no TDE, houve diferenças nos três subtestes que compõem o instrumento e no escore total, fazendo-se notar, em todas as medidas, melhor desempenho alcançado pelo grupo controle. A estimativa da magnitude de efeito foi classificada como elevada em todas as medidas, como pode ser visualizado na Tabela 2. 
Tabela 2.

Distribuição de frequência de classificações e comparação das médias do TDE entre os grupos.

\begin{tabular}{|c|c|c|c|}
\hline \multirow{3}{*}{$\begin{array}{c}\text { TDE } \\
\text { classificações }\end{array}$} & \multicolumn{2}{|c|}{ Grupos } & \multirow{3}{*}{$\mathrm{p}$-valor ${ }^{\mathrm{a}}$} \\
\hline & GD & GC & \\
\hline & $f(\%)$ & $f(\%)$ & \\
\hline \multicolumn{4}{|l|}{ Escrita } \\
\hline Inferior & $10(91)$ & $2(22)$ & \multirow{3}{*}{$0,01 *$} \\
\hline Média & $1(9)$ & $5(56)$ & \\
\hline Superior & 0 & $2(22)$ & \\
\hline \multicolumn{4}{|l|}{ Aritmética } \\
\hline Inferior & $11(100)$ & $8(89)$ & \multirow{2}{*}{0,45} \\
\hline Média & 0 & $1(11)$ & \\
\hline \multicolumn{4}{|l|}{ Leitura } \\
\hline Inferior & $11(100)$ & $3(33)$ & \multirow{3}{*}{$<0,001^{*}$} \\
\hline Média & 0 & $5(56)$ & \\
\hline Superior & 0 & $1(11)$ & \\
\hline \multicolumn{4}{|l|}{ Total } \\
\hline Inferior & $11(100)$ & $6(67)$ & \multirow{3}{*}{0,07} \\
\hline Média & 0 & $2(22)$ & \\
\hline Superior & 0 & $1(11)$ & \\
\hline Subtestes & $M(D P)$ & $M(D P)$ & p-valor $;$ Effect Size \\
\hline Escrita & $12,45(10,01)$ & $28,33(4,09)$ & $<0,001 * ; \mathrm{ES}=-0,72$ \\
\hline Aritmética & $10,27(3,80)$ & $17,11(2,62)$ & $<0,001^{*} ; \mathrm{ES}=-0,72$ \\
\hline Leitura & $38,82(25,03)$ & $66,00(3,16)$ & $<0,001^{*} ; \mathrm{ES}=-0,61$ \\
\hline Total & $61,55(36,76)$ & $110,33(7,66)$ & $<0,001 * ; \mathrm{ES}=-0,68$ \\
\hline
\end{tabular}

Nota: GD - grupo com queixas de dificuldades de aprendizagem; GC - grupo controle; f - Frequência; \% - Porcentagem; ES - Effect size; a - Teste Exato de Fisher; b - Teste de Mann-Whitney; c - Cohen's d; *valor significativo.

Quando consideradas as comparações entre os grupos no TISD (Tabela 3), verificamos que houve diferenças estatisticamente significativas nos seguintes escores: Leitura $(p \leq 0,01)$, Escrita $(p \leq 0,01)$, Cálculo $(p \leq 0,01)$, Consciência Fonológica $(p \leq 0,03)$ e Memória de Trabalho $(p \leq 0,01)$. Em todos os casos o GD apresentou médias mais altas quando comparado com o GC. Os grupos não se diferenciaram nos escores nos subtestes de Atenção Visual, Habilidade Motora e em Nomeação Rápida, devendo-se salientar o fato de que, ainda que as diferenças não tenham se mostrado significativas, nessas medidas o GD também apresenta médias mais altas que o GC. Por meio da estimativa de magnitude de 
efeito (Cohen's d) as diferenças foram consideradas pequenas (Atenção Visual, Habilidade Motora e Nomeação Rápida) e médias (Leitura, Escrita, Consciência Fonológica e Memória de Trabalho).

Por fim, com a finalidade de busca por evidências baseadas nas relações com variáveis externas, foram realizadas correlações entre os escores do TDE e do TISD, considerando-se a amostra total (Tabela 4). Os resultados mostraram que os subtestes do TISD (Leitura, Escrita, Cálculo, Consciência Fonológica, Nomeação Rápida, Memória de Trabalho) e escore total mostraram correlações negativas e estatisticamente significativas com os escores do TDE (Escrita, Aritmética, Leitura e Total), variando entre -0,45 e -0,85, consideradas moderadas e altas. Não foram verificadas correlações significativas entre os subtestes de Atenção Visual e Habilidade Motora do TISD com nenhum dos subtestes do TDE e sua pontuação total. Notadamente em relação ao total do TISD e total do TDE, a correlação mostrou-se alta $(r=-0,86)$.

\section{DISCUSSÃO}

As análises realizadas para o Teste de Desempenho Escolar indicaram melhor desempenho do GC em relação ao GD em todos os subtestes (Escrita, Aritmética e Leitura) e no escore total. A estimativa da magnitude de efeito corrobora com tais resultados, sendo classificada como elevada. Vale ressaltar que o estudo pretendeu comparar o desempenho de crianças com dificuldades de aprendizagem e crianças sem dificuldades. Por este motivo era esperado desempenho mais baixo em todos os subtestes do TDE no GD quando comparado ao GC, resultado que foi confirmado.

Na comparação do desempenho do grupo com queixas de dificuldades de aprendizagem e sem dificuldades de aprendizagem no segundo instrumento (TISD) verificamos diferenças estatisticamente significativas nos escores nos subtestes de Leitura, Escrita, Cálculo, Consciência Fonológica e Memória de Trabalho e ausência de diferenças em Atenção Visual, Habilidade Motora e Nomeação Rápida e no Total. 
Tabela 3.

Comparação das médias dos escores do TISD entre os grupos.

\begin{tabular}{|c|c|c|c|c|}
\hline \multirow{2}{*}{ TISD } & GD & GC & \multirow{2}{*}{$\mathrm{p}$-valor ${ }^{\mathrm{a}}$} & \multirow{2}{*}{ Effect-size ${ }^{b}$} \\
\hline & $M(D P)$ & $M(D P)$ & & \\
\hline L1 & $0,36(0,67)$ & $0,11(0,33)$ & 0,35 & 0,23 \\
\hline L2 & $2,82(3,49)$ & $0,00(0,00)$ & $0,01 *$ & 0,50 \\
\hline L3 & $4,00(2,90)$ & $1,22(0,97)$ & $0,01 *$ & 0,54 \\
\hline Leitura Total & $7,18(6,60)$ & $1,33(1,22)$ & $0,01 *$ & 0,52 \\
\hline $\mathrm{E} 1$ & $0,45(0,69)$ & $0,56(0,73)$ & 0,73 & $-0,08$ \\
\hline E2 & $4,82(2,79)$ & $2,56(1,74)$ & $0,05 *$ & 0,44 \\
\hline E3 & $6,45(1,57)$ & $3,78(0,83)$ & $0,00 *$ & 0,73 \\
\hline Escrita Total & $11,73(4,36)$ & $6,89(2,47)$ & $0,01 *$ & 0,56 \\
\hline AV1 & $0,00(0,00)$ & $0,33(1,00)$ & 0,27 & $-0,23$ \\
\hline $\mathrm{AV} 2$ & $2,91(2,07)$ & $2,11(1,05)$ & 0,53 & 0,24 \\
\hline Atenção Visual Total & $2,91(2,07)$ & $2,44(1,67)$ & 0,66 & 0,12 \\
\hline $\mathrm{C} 1$ & $0,09(0,30)$ & $0,00(0,00)$ & 0,37 & 0,21 \\
\hline C2 & $0,09(0,30)$ & $0,11(0,33)$ & 0,88 & $-0,03$ \\
\hline C3 & $0,73(0,47)$ & $0,22(0,44)$ & $0,03 *$ & 0,49 \\
\hline $\mathrm{C} 4$ & $0,82(0,40)$ & $0,11(0,33)$ & $0,00 *$ & 0,70 \\
\hline Cálculo Total & $1,73(1,01)$ & $0,44(0,73)$ & $0,01 *$ & 0,59 \\
\hline HM1 & $0,64(0,50)$ & $0,44(0,73)$ & 0,32 & 0,16 \\
\hline HM2 & $0,73(0,47)$ & $1,00(0,71)$ & 0,34 & $-0,22$ \\
\hline HM3 & $1,55(0,69)$ & $1,11(0,93)$ & 0,27 & 0,26 \\
\hline HM4 & $0,64(0,81)$ & $0,56(0,53)$ & 1,00 & 0,06 \\
\hline HM5 & $0,55(0,52)$ & $0,33(0,50)$ & 0,36 & 0,21 \\
\hline Habilidade Motora Total & $4,09(1,64)$ & $3,44(2,19)$ & 0,46 & 0,17 \\
\hline CF1 & $1,36(0,81)$ & $0,89(0,93)$ & 0,26 & 0,26 \\
\hline CF2 & $2,73(0,47)$ & $1,67(1,12)$ & $0,01 *$ & 0,53 \\
\hline Consciência Fonológica Total & $4,09(1,04)$ & $2,56(1,51)$ & $0,03 *$ & 0,51 \\
\hline NR1 & $9,73(10,00)$ & $4,00(1,73)$ & 0,09 & 0,37 \\
\hline NR2 & $4,73(2,33)$ & $3,22(0,83)$ & 0,13 & 0,40 \\
\hline Nomeação Rápida Total & $14,45(11,60)$ & $7,22(2,05)$ & 0,07 & 0,40 \\
\hline MT1 & $2,64(0,81)$ & $1,56(1,13)$ & $0,03 *$ & 0,48 \\
\hline MT2 & $2,64(0,92)$ & $1,89(0,93)$ & 0,11 & 0,38 \\
\hline Memória de Trabalho Total & $5,27(1,01)$ & $3,44(1,51)$ & $0,01 *$ & 0,58 \\
\hline Total Geral & $51,55(22,78)$ & $27,44(5,66)$ & $0,00 *$ & 0,59 \\
\hline
\end{tabular}

Nota: GD - grupo com queixas de dificuldades de aprendizagem; GC - grupo controle; M - Média; DP - Desvio Padrão; L - Leitura; E - Escrita; AV - Atenção visual; C - Cálculo; HM - Habilidade motora; CF - Consciência fonológica; NR Nomeação rápida; MT - Memória de trabalho; a - Teste de Mann-Whitney; b Cohen's d; *valor significativo. 
Tabela 4.

Correlação entre as pontuações do TDE e do TISD (coeficiente de correlação de Spearman - r).

\begin{tabular}{ccccc}
\hline $\begin{array}{c}\text { Sustestes TISD/ } \\
\text { TDE }\end{array}$ & $\begin{array}{c}\text { Escrita } \\
(\text { TDE })\end{array}$ & $\begin{array}{c}\text { Aritmética } \\
(\text { TDE })\end{array}$ & $\begin{array}{c}\text { Leitura } \\
(\text { TDE) }\end{array}$ & Total (TDE) \\
\hline Leitura & $-0,744^{* *}$ & $-0,616^{* *}$ & $-0,706^{* *}$ & $-0,781^{* *}$ \\
Escrita & $-0,888^{* *}$ & $-0,591^{* *}$ & $-0,761^{* *}$ & $-0,858^{* *}$ \\
Atenção Visual & $-0,136$ & $-0,263$ & $-0,021$ & $-0,141$ \\
Cálculo & $-0,481^{*}$ & $-0,732^{* *}$ & $-0,511^{*}$ & $-0,586^{* *}$ \\
Habilidade Motora & $-0,002$ & $-0,246$ & $-0,024$ & $-0,160$ \\
Consciência & $-0,622^{* *}$ & $-0,450^{*}$ & $-0,576^{* *}$ & $-0,574^{* *}$ \\
Fonológica & $-0,628^{* *}$ & $-0,442$ & $-0,700^{* *}$ & $-0,609^{* *}$ \\
Nomeação Rápida & $-0,806^{* *}$ & $-0,710^{* *}$ & $-0,754^{* *}$ & $-0,800^{* *}$ \\
Memória de Trabalho & $-0,838^{* *}$ & $-0,747^{* *}$ & $-0,747^{* *}$ & $-0,869^{* *}$ \\
Total &
\end{tabular}

*valor significativo a $\mathrm{p}<0,05 ; * *$ valor significativo a $\mathrm{p}<0,01$.

Considerando-se cada habilidade isoladamente, pode-se verificar que, no desempenho da leitura houve diferença significativa entre o GD e o GC, tanto no subteste do TDE quanto nos subtestes de leitura de palavras, leitura de pseudopalavras e no total do TISD, mas não na identificação de letras do alfabeto. Tal achado vai de encontro ao afirmado por Fletcher et al. (2009) de que as dificuldades mais significativas apresentadas por indivíduos com DD estariam relacionadas com a leitura da palavra como um todo, apesar de também apresentarem dificuldades na aprendizagem do sistema alfabético (Carvalhais, 2010). Frequentemente, estudos evidenciam que a leitura depende da decodificação via rota fonológica, na qual os grafemas que compõem a palavra devem ser interpretados pelas possibilidades de fonemas que podem representar, e também via rota lexical, na qual as palavras são reconhecidas visualmente, permitindo uma leitura mais rápida, automática e fluente (Hawelka, Gagl \& Wimmer, 2010; Shaywitz \& Shaywitz, 2005; Ziegler et al., 2008). O atraso ou comprometimento decorrentes de déficits nos processamento cognitivos que compõem essas rotas explicariam as dificuldades na leitura de palavras, como ocorre nos casos de DD. No presente caso, verificou-se que somente a leitura de letras do alfabeto não foi sensível para discriminação entre ambos os grupos. Além disto, a magnitude de efeito mostrou-se pequena para este subteste. 
Como ocorreu com o subteste de leitura em relação às letras, no subteste de escrita houve diferenças significativas entre os grupos no ditado de palavras, ditado de pseudopalavras e no total, mas não no ditado de letras no TISD. A literatura mostra que crianças com sinais de DD possuem baixa proficiência tanto na decodificação quanto na codificação de palavras, provavelmente em razão dos déficits nas rotas supracitadas (Bergmann \& Wimmer, 2008; Wimmer et al., 2010), situação que foi confirmada na presente pesquisa.

No subteste de atenção visual não foram encontradas diferenças entre os grupos GD e GC, situação que vai de encontro aos achados da literatura nacional (Lima, Salgado-Azoni, \& Ciasca, 2013) e internacional sobre crianças com DD (Facoetti et al., 2010). Isso porque alguns modelos teóricos e achados empíricos indicam que indivíduos com DD apresentam baixo desempenho em tarefas de atenção visual e que tais dificuldades são tão importantes quanto as dificuldades no processamento fonológico da linguagem (Heim et al., 2010; Lobier, Zoubrinetzky \& Valdois, 2012). Outro dado importante refere-se ao fato de que frequentemente instrumentos internacionais para avaliação da DD apresentam subtestes para avaliar a atenção, sendo considerada uma habilidade prejudicada nesse transtorno (Lima et al., 2011; Lima et al., 2013). Este aspecto não foi confirmado pelo presente estudo, devendo-se, entretanto, ressaltar a necessidade de cautela na interpretação dos dados, uma vez que a amostra do GD apresentava queixas de dificuldades de aprendizagem e não diagnóstico de DD.

Quanto ao subteste cálculo, foram verificadas diferenças significativas entre ambos os grupos no resultado total. Observa-se que não houve diferenças nos itens de adição e subtração, ao contrário do que houve com os subtestes de multiplicação e divisão. Vale lembrar que o GD aqui investigado foi composto por crianças com dificuldades globais no aprendizado, dentre as quais poderia abrangeria também dificuldades em aritmética, as quais foram, parcialmente, confirmadas. Considerando o objetivo do TISD, que é a identificação de sinais de $D D$, espera-se que, futuramente, novos estudos de busca por evidências de validade de critério em relação a esse grupo, contemplando número maior de participantes, sejam observados outros resultados, mais favoráveis, não confirmando a existência de diferenças de desempenho nos indivíduos com dislexia quando comparados a grupos controles. Isso porque, o raciocínio lógico usualmente mostra-se preservado nos indivíduos com DD (Caldonazzo et al., 
2006), desde que a atividade não envolva o reconhecimento/leitura de algoritmos, por meio do seu envolvimento com conteúdos verbais e a leitura do próprio problema (Caldonazzo et al., 2006; Simmons \& Singleton, 2008), como as do TISD.

No subteste habilidade motora também não houve diferença estatisticamente significativa entre os grupos. Tal achado confirma os resultados que vêm sendo apresentados em alguns estudos nacionais, os quais verificaram baixas correlações entre o baixo desempenho acadêmico e prejuízos em algumas habilidades motoras, tais como função motora fina, grossa e sensorial, evidenciando que as dificuldades em habilidades motoras podem ocorrer tanto em crianças com dificuldades e transtornos de aprendizagem e mesmo naquelas sem dificuldades escolares, não sendo este um fator determinante para alguns desses casos (Capellini, Coppede, \& Valle, 2010; Coppede, Okuda, \& Capellini, 2012). Por outro lado, pensando especificamente em um grupo com DD, em um estudo nacional foi verificada alteração nas funções visomotoras por meio do "Teste Visomotor de Bender", como: dificuldade em fechamento, prejuízos em coordenação motora e dificuldade em angulação (Santos \& Jorge, 2007), compatível aos achados internacionais de prejuízos motores em indivíduos com DD (Nicolson \& Fawcett, 2011). Nesse sentido, novamente espera-se que resultados diferentes sejam encontrados por ocasião de aplicações futuras do TISD em indivíduos com DD.

Em relação ao subteste de consciência fonológica, o presente estudo verificou que o desempenho das crianças do grupo com dificuldades de aprendizagem mostrou-se significativamente pior do que 0 grupo sem dificuldades. Pesquisas em tarefas linguísticas evidenciaram relação entre o déficit da consciência fonológica e dificuldades na escrita em crianças com fracasso escolar (Deuschle \& Cechella, 2009; Salgado, 2010). Provavelmente existiria um atraso do desenvolvimento dessas funções cognitivo-linguísticas em crianças que apresentam dificuldades de leitura e escrita (Bergmann \& Wimmer, 2008; Rapcsak et al., 2009; Carvalhais, 2010), fato que foi confirmado na presente pesquisa, em indivíduos com sinais indicativos de dificuldades de aprendizagem, fazendo-se uso de subteste do TISD desenvolvido especificamente para a avaliação dessa habilidade. Novos dados, coletados junto a amostra de indivíduos com DD poderão trazer dados acerca do comportamento desse subteste nessa população específica. 
Considerando os subtestes do TISD que avaliam consciência fonológica, identificação (CF1) e produção de rimas (CF2), houve diferença significativa entre os grupos somente na produção de rima. Porém, o CF1 também apresentou magnitude de feito moderada. Em um estudo realizado por Germano, Pinheiro e Capellini (2009) também foi encontrado esse padrão quando crianças com DD e crianças sem dificuldades escolares foram comparadas. Os resultados mostraram pior desempenho em produção do que em identificação de rima no primeiro grupo quando comparado ao segundo, corroborando os achados do presente estudo, com amostra composta por indivíduos com TA. Salgado (2010) descreveu a importância de se diferenciar as dificuldades na produção de rima quando comparadas à identificação, já que este é um importante componente em um processo de avaliação e direcionamento da intervenção para crianças com DD. Estudos com intervenção precoce para crianças de risco para DD também preconizam atividades de consciência fonológica, nas quais muitas delas são a identificação e produção de rimas (Deuschle \& Cechella, 2009; Murphy \& Schochat, 2009), de modo a confirmar a importância do subteste em questão, assim como da busca por evidências de validade junto a população para o qual foi construído.

Em nomeação rápida não foram verificadas diferenças significativas intragrupo, mas a estimativa de magnitude de efeito indicou diferenças elevadas nos dois subtestes e total, com prejuízos maiores no GD. Comumente na literatura é verificado maior tempo e frequência de erros na realização desse tipo de tarefa em crianças com dificuldades e TA quando comparadas àquelas sem dificuldades escolares, uma vez que essa tarefa avalia a velocidade de acesso ao léxico (Silva, Cunha, Pinheiro \& Capellini, 2012; Ziegler, Pech-Georgel, Dufau \& Grainger, 2010). Nesse sentido, os resultados do estudo exploratório aqui apresentado não se mostraram favoráveis ao subteste criado, ainda que a limitação no número de participantes provavelmente tenha exercido influência importante nos resultados obtidos. Estudos com amostras maiores e mais representativas poderão confirmar ou não a capacidade discriminativa do subteste em questão.

No subteste de memória de trabalho houve diferença estatisticamente significativa entre os dois grupos avaliados em dígitos e no total, mas não em pseudopalavras, apesar de todos os escores terem sido maiores para o GD. Porém, a estimativa da magnitude de efeito indicou diferença elevada na 
memória de trabalho para pseudopalavras. A literatura científica têm apontado para o fato de que prejuízos em memória de trabalho são comuns em indivíduos com DD (Lukasova, Barbosa \& Macedo, 2009; Menghini, Finzi, Carlesimo \& Vicari, 2011), de modo que o resultado demonstrado no presente subteste do TISD também evidenciou prejuízo em indivíduos com indicativos de TA. Tal resultado evidencia a hipótese de que crianças com dificuldade de aprendizagem que também apresentam alteração na memória de trabalho podem apresentar risco para a DD, de maneira a justificar a avaliação dessa habilidade durante o processo de diagnóstico para esse quadro.

No escore total do TISD houve diferença significativa entre os grupos, sendo que o GD apresentou escores mais altos que o GC, a qual poderia estar indicando a presença de dificuldades nas habilidades cognitivas que fazem parte do teste. Os resultados podem sugerir também que, neste estudo piloto, o TISD mostrouse sensível para detectar diferenças de desempenho entre crianças com dificuldades de aprendizagem daquelas sem dificuldades de aprendizagem nas habilidades englobadas no instrumento.

Quando correlacionados os escores do TDE ao TISD, foram verificadas correlações negativas e significativas, consideradas moderadas e altas. Tais resultados eram esperados, visto que ambos os instrumentos avaliam construtos semelhantes ou relacionados às habilidades escolares. Ressalta-se que os resultados apresentam valores negativos pois os resultados de ambos os testes são inversamente proporcionais, lembrando que quanto mais pontos obtidos no TDE, melhor o desempenho acadêmico, ao contrário do que se espera em relação no TISD, cujo aumento na pontuação aponta para um maior número de dificuldades. Notadamente foram observadas correlações negativas, estatisticamente significativas e altas entre subtestes do TISD que avaliam as mesmas habilidades que o TDE, como Leitura $(r=-0,70)$, Escrita $(r=-0,88)$ e Cálculo/Aritmética $(r=-0,73)$. Também devemos ressaltar as correlações negativas significativas e altas entre o escore total do TISD e todos os escores do TDE. Desta maneira os resultados permitem afirmar que o TISD mostrou-se relacionado com outro teste já padronizado para a população brasileira e que mede construto semelhante, de modo que, ainda que em estudo exploratório inicial, as evidências de validade com medidas externas foram confirmadas.

Somente os subtestes de Atenção Visual e Habilidade Motora apresentaram correlações não significativas com o TDE. Apesar da necessidade destas funções 
(atenção e habilidade motora) para a leitura, escrita e cálculo, talvez estas relações sejam menos evidentes que aquelas encontradas em relação à consciência fonológica, memória de trabalho e nomeação rápida (habilidades que fazem parte do processamento fonológico). Desta maneira, pode existir uma justificativa teórica e empírica para a baixa correlação encontrada. Por outro lado, estudos futuros com o TISD devem reconsiderar os subtestes de Atenção Visual e Habilidade Motora, principalmente no que se refere ao sistema de pontuação.

\section{CONSIDERAÇÕES FINAIS}

Com base nos objetivos estabelecidos para o estudo, verificou-se que os escores do TISD foram diferentes para crianças com e sem dificuldades de aprendizagem, de modo que as primeiras obtiveram pior desempenho, conforme hipotetizado durante o processo de construção do instrumento. O TISD também mostrou-se correlacionado com os escores do TDE, instrumento já padronizado para avaliação do desempenho escolar. Dessa maneira foram encontradas evidências de validade do TISD para diferenciação de grupos e correlação com variáveis externas, consistindo em evidências favoráveis para a continuidade dos estudos de seus critérios psicométricos.

Estudos posteriores com o TISD devem buscar outras evidências de validade, dado que o número reduzido de participantes na presente pesquisa impossibilita que os resultados encontrados sejam generalizados para a população. Sugere-se que novos estudos e que contemplem amostras maiores e específicas para o quadro foco do instrumento (Dislexia do Desenvolvimento), de modo que sua validade para identificação do mesmo possa ser confirmada.

\section{REFERÊNCIAS}

Alves, R. J. R., \& Nakano, T. C. (no prelo). Criatividade em indivíduos com transtornos e dificuldades de aprendizagem: Revisão de pesquisas. Psicologia Escolar e Educacional.

Alves, R. J. R. (2011). Teste de identificação de sinais de Dislexia (TISD). Monografia de Conclusão de Curso de Especialização, Universidade Estadual de Campinas, São Paulo. 
Alves, R. J. R., Lima, R. F., Salgado-Azoni, C. A., Carvalho, M. C., \& Ciasca, S. M. (no prelo). Teste de Identificação de Sinais de Dislexia (TISD): Processos de construção. Estudos de Psicologia (PUC-Campinas).

American Psychiatric Association (APA) (2002). Manual diagnóstico e estatístico de transtornos mentais (DSM-IV-TR). Porto Alegre: Artes Médicas.

American Psychological Association (APA) (1999). Standards for educational and psychological testing. Washington, DC.

Anastasi, A., \& Urbina S. (2000). Testagem psicológica. Porto Alegre: Artes Médicas.

Bergmann, J., \& Wimmer, H. (2008). A dual-route perspective on poor reading in a regular orthography: Evidence from phonological and orthographic lexical decisions. Cognitive Neuropsychology, 25(5), 653-676.

Caldonazzo, A., Salgado, C. A., Capellini, A. S., \& Ciasca, S. M. (2006). Desempenho na resolução de problemas envolvendo o conceito aditivo em sujeitos com Dislexia do Desenvolvimento. Revista Psicopedagogia, 23(71), 116-123.

Capellini, S. A., Coppede, A. C., \& Valle, T. R. (2010). Função motora fina de escolares com dislexia, distúrbio e dificuldades de aprendizagem. Pró-fono Revista de Atualização Científica, 22(3), 201-208.

Capellini, S. A., Sampaio, M. N., Matsuzawa, M. T., Oliveira, A. M., Fadini, C. C., \& Martins, M. A. (2009). Protocolo de identificação precoce dos problemas de leitura: estudo preliminar com escolares de $1^{0}$ ano escolar. Psicopedagogia, 26(81), 367-375.

Capovilla, A. G. S., Smythe, I., Capovilla, F. C., \& Everatt, J. (2001). Adaptação brasileira do International Dyslexia Test: Perfil cognitivo de crianças com escrita pobre. Temas sobre Desenvolvimento, 10(57), 30-37.

Carvalhais, L. S. A. (2010). Construção de instrumentos de avaliação da dislexia. Tese de Doutorado, Universidade de Aveiro: Aveiro.

Carvalho, F. B., Crenitte, P. A. P., \& Ciasca, S. M. (2007). Distúrbios de aprendizagem na visão do professor. Psicopedagogia, 24(75), 229-239.

Conboy, J. E. (2003). Algumas medidas típicas univariadas de magnitude do efeito. Análise Psicológica, 21(2), 145-158. 
Coppede, A. C., Okuda, P. M. M., \& Capellini, S. A. (2012). Desempenho de escolares com dificuldades de aprendizagem em função motora fina e escrita. Revista Brasileira de Crescimento e Desenvolvimento Humano, 22(3), 297-306.

Cox, E. A. (2002). An evaluation of the psychometric properties of the test of dyslexia e dysgraphia. Tese de Doutorado. University of Tennessee: EUA.

Dancey, C., \& Reidy, J. (2006). Estatística sem matemática para Psicologia: Usando SPSS para Windows. Porto Alegre: Artmed.

Deuschle, V. P., \& Cechella, C. (2009). O déficit em consciência fonológica e sua relação com a dislexia: Diagnóstico e intervenção. Revista CEFAC, 11(Supl 2), 194-200.

Eden, G. F., \& Zeffiro, T. A. (1998). Neural systems affected in developmental dyslexia revealed by functional neuroimaging. Neuron, 21(2), 279-282.

Facoetti, A., Trussardi, A. N., Ruffino, M., Lorusso, M.L., Cattaneo, C., Galli, R., Molteni, M., \& Zorzi, M. (2010). Multisensory spatial attention deficits are predictive of phonological decoding skills in developmental dyslexia. Journal of Cognitive Neuroscience, 22(5), 1011-1025.

Fletcher, J. M., Lyons, G. R., Fuchs, L. S., \& Barnes, M. A. (2009). Transtornos de aprendizagem: Da identificação à intervenção. Porto Alegre: Artmed.

Fuchs, L. S., \& Fuchs, D. (1998). Treatment validity: A simplifying concept for reconceptualizing the identification of learning disabilities. Learning Disabilities Research and Practice, 4, 204-219.

Germano, G. D., \& Capellini, S. A. (2011). Desempenho de escolares com dislexia, transtornos e dificuldades de aprendizagem em provas de habilidades metafonológicas (PROHFON). Jornal da Sociedade Brasileira de Fonoaudiologia, 23(2), 135-141.

Germano, G. D., Pinheiro, F. H., \& Capellini, S. A. (2009). Desempenho de escolares com Dislexia do Desenvolvimento em tarefas fonológicas e silábicas. Revista CEFAC, 11(2), 213-220.

Grigorenko, E.L. (2005). A conservative meta-analysis of linkage-association studies of developmental dyslexia. Scientific Studies of Reading, 9(3), 285316.

Hawelka, S., Gagl, B., \& Wimmer, H. (2010). A dual-route perspective on eye movements of dyslexic readers. Cognition, 115(3), 367-279. 
Heim, S., Grande, M., Pape-Neumann, J., van Ermingen, M., Meffert, E., Grabowska, A., Huber, W., \& Amunts, K. (2010). Interaction of phonological awareness and 'magnocellular' processing during normal and dyslexic reading: behavioural and fMRI investigations. Dyslexia, 16(3), 258-282.

Lima, R. F. (2011). Sintomas depressivos e funções cognitivas em crianças com dislexia do desenvolvimento. Dissertação de Mestrado, Faculdade de Ciências Médicas, Universidade Estadual de Campinas, São Paulo.

Lima, R. F., Salgado, C. A., \& Ciasca, S. M. (2011). Attentional performance and executive functions in children with learning difficulties. Psicologia: Reflexão e Crítica, 24(4), 685-691.

Lima, R. F., Salgado-Azoni, C. A. S, \& Ciasca, S. M. (2013). Attentional and executive deficits in Brazilian children with developmental dyslexia. Psychology, 4(10A):1-6.

Lobier, M., Zoubrinetzky, R., \& Valdois, S. (2012). The visual attention span deficit in dyslexia is visual and not verbal. Cortex, 48(6), 768-773.

Lukasova, K., Barbosa, A. C. C., \& Macedo, E. C. D. (2009). Discriminação fonológica e memória em crianças com dislexia e bons leitores. PsicoUSF, 14(1), 1-9.

Menghini, D., Finzi, A., Carlesimo, G. A., \& Vicari, S. (2011). Working memory impairment in children with developmental dyslexia: is it just a phonological deficity? Developmental Neuropsychology, 36(2), 199-213.

Murphy, C. F. B., \& Schochat, E. (2009). Correlações entre leitura, consciência fonológica e processamento temporal auditivo. Pró-Fono, 21(1), 13-18.

Nicolson, R. I., \& Fawcett, A. J. (2003). Sample report of Dyslexia Early Screening Test - Second edition (DEST-2). Universidade de Sheffield: Inglaterra.

Nicolson, R. I., \& Fawcett, A. J. (2011). Dyslexia, dysgraphia, procedural learning and the cerebellum. Cortex, 47(1), 117-127.

Nunes, C. H. S. S., \& Primi, R. (2010). Aspectos técnicos e conceituais da ficha de avaliação dos testes psicológicos. Avaliação psicológica: Diretrizes na regulamentação da profissão (pp. 101-128). Brasília: Conselho Federal de Psicologia.

Organização Mundial de Saúde (OMS). (2008). CID-10: Classificação de transtornos mentais e de comportamento: Descrições clínicas e diretrizes diagnósticas. Porto Alegre: Artes Médicas. 
Plomin, R., \& Kovas, Y. (2005). Generalist genes and learning disabilities. Psychological Bulletin, 131(4), 592-617.

Rapcsak, S. Z., Beeson, P. M., Henry, M. L., Leyden, A., Kim, E., Rising, K., Andersen, S., \& Cho, H. (2009). Phonological dyslexia and dysgraphia: cognitive mechanisms and neural substrates. Cortex, 45(5), 575-591.

Salgado, C.A. (2010). Programa de remediação fonológica de leitura e escrita em crianças com Dislexia do Desenvolvimento. Tese de Doutorado. Universidade Estadual de Campinas, São Paulo.

Santos, A. A. A., \& Jorge, L. M. (2007). Teste de Bender com disléxicos: Comparação de dois sistemas de pontuação. Psico-USF, 12(1), 13-21.

Shaywitz, S. E., \& Shaywitz, B. A. (2005). Dyslexia (specific reading disability). Biological Psychiatry, 57(11), 1301-1309.

Shaywitz, S. E., \& Shaywitz, B. A. (2008). Paying attention to reading: The neurobiology of reading and dyslexia. Development and psychopathology, 20(04), 1329-1349.

Siegel, L .S. (2003). Basic cognitive processes and reading disabilities. In Swanson, H.L., Harris, K.R. \& Graham, S. Handbook of learning disabilities (pp. 158-181). New York: Guilford Press.

Silva, C., Cunha, V. L. O., Pinheiro, F. H., \& Capellini, S. A. (2012). Nomeação rápida, leitura e compreensão em escolares com dificuldades de aprendizagem. Jornal da Sociedade Brasileira de Fonoaudiologia, 24(4), 355-360.

Simmons, F. R., \& Singleton, C. (2008). Do weak phonological representations impact on arithmetic development? A review of research into arithmetic and dyslexia. Dyslexia, 14(2), 77-94.

Stein, L. M. (1994). Teste de desempenho escolar. São Paulo: Casa do Psicólogo.

Swanson, H. L., Harris, K., \& Graham, S. (Eds.) (2003). Handbook of learning disabilities. New York: Guilford Press.

Wimmer, H., Schurz, M., Sturm, D., Richlan, F., Klackl, J., Kronbichler, M., \& Ladurner, G. (2010). A dual-route perspective on poor reading in a regular orthography: An fMRI study. Cortex, 46(10), 1284-1298.

Wong, B., \& Butler, D. (2012). Learning about learning disabilities. California: Elsevier. 
Ziegler, J. C., Castel, C., Pech-Georgel, C., George, F., Alario, F., \& Perry, C. (2008). Developmental dyslexia and the dual route model of reading: Simulating individual differences and subtypes. Cognition, 107(1), 151-178.

Ziegler, J. C., Pech-Georgel, C., Dufau, S., \& Grainger, J. (2010). Rapid processing of letters, digits and symbols: what purely visual-attentional deficit in developmental dyslexia?. Developmental science, 13(4), F8-F14.

Contato: rauniroama@gmail.com

Recebido em: 10/02/2014

Revisado em: 28/02/2014

Aceito em: $10 / 03 / 2014$ 